\title{
A GPS APPLICATION FOR POSITIONING OF EARTHWORK EQUIPMENTS
}

Tatsunori Sada, Yoshifumi Nakagawa, Tomonori Takada, Technical Research Institute, Mitsui Construction Co., Ltd.

518-1 Komaki, Nagareyama-shi, Chiba, 270-01, Japan

\section{ABSTRACT}

GPS is a new radio-wave positioning system, which allows the user to ascertain his position from waves transmitted from the GPS satellites. A system using GPS navigation sensors was applied to positioning of earthwork equipment. While the precision obtained in single-point positioning was only around $20 \mathrm{~m}$, the precision could be reduced to around $2 \mathrm{~m}$ in differential positioning using two systems, precision that would allow the system to distinguish between loci of earthwork equipment.

\section{INTRODUCTION}

GPS (Global Positioning System) is a new space-based radio positioning and surveying system that is being developed by the U.S. Department of Defence. The user can ascertain his position (latitude, longitude and altitude) from the radio waves, which are sent out from the GPS satellites and which he receives through special sensors. There are two types of GPS sensors, namely the navigation sensors, which are less accurate but allow the users to ascertain their position in real time, and the geodetic sensors, which are more accurate but require off-line processing. The former are used for navigation of airplanes, ships and motor vehicles. The latter allows the user to pinpoint his position to within $1 \mathrm{~cm}$ and research is beginning to be conducted on its application to surveying and to civil engineering work, for example, in measurement of ground deformation. ${ }^{\prime \prime}$

The authors have been conducting research on the possibility of applying both the navigation and geodetic sensors to engineering work from the point of view of precision and facility of operation. ${ }^{2}$ Discussions are made in the present paper on the navigation sensors in particular, with a view to developing a system for real time positioning with sensors installed on moving earthwork equipment (vibratory rollers, bulldozers etc.), as a possible application of the sensors to on-site monitoring for machine operation. Discussed below is the position indication system using GPS navigation sensors, together with the results of the test on the operational characteristics of the system and the test in which the sensors were installed on a vibratory roller.

\section{OUTLINE OF GPS}

\subsection{Composition of GPS}

The GPS consists of the space, control and user segments (Figure 1).

The space segment is the GPS satellites themselves, which orbit the earth at a height of approximately $20,000 \mathrm{~km}$. By the time the system is completed, there will be 21 such satellites (plus 3 reserve satellites) in orbit, ensuring that there will be at least 4 satellites in view from any given point on earth. Fifteen satellites have been launched as of March 1991.

Two bands of radio waves, referred to as the L1 $(1.6 \mathrm{GHz})$ and L2 $(1.2 \mathrm{GHz})$ bands, are constantly transmitted from the GPS satellites for positioning purposes. The L1 band contains two types of pseudo random noise codes, called the $\mathrm{C} / \mathrm{A}$ and $\mathrm{P}$ codes, while the L2 band contains the $\mathrm{P}$ code. Since the $\mathrm{P}$ code is used for confidential military messages, the $\mathrm{C} / \mathrm{A}$ code only can be used by ordinary users. 
The control segment includes the tracking and control system for the satellites. Its main functions are tracking the satellite orbits, checking for errors on the atomic clocks on the satellites, renewal of various positioning calculation data transmitted to users from the satellites and correcting the satellite orbits.

The user segment consists of the sensors used for positioning. The two types of navigation and geodetic sensors involve different mechanisms and analysis processes. Civilian firms are free to develop sensors and users need only have the appropriate sensors, aerials and computers to use the GPS in any place.

\subsection{Principles of Positioning by GPS Navigation Sensors}

The position of the sensor can be ascertained if there are three satellites and their positions are known, together with their distances from the sensor. The positions of the GPS satellites at any given time at which the user wishes to ascertain his position can be calculated accurately from the orbit data transmitted from the satellites. The difference between the time at which the radio waves leave the satellites and that at which they are received will give the transmission time, which one has to multiply by the speed of light to obtain one's distance from the satellite. The clock in the user sensor, however, is not necessary accurate and the transmission time thus measured and consequently the distance so calculated may be inaccurate. The distance thus measured is referred to as the pseudo range. A fourth satellite is required in order to measure the distances accurately. The computer in the sensor will make corrections to the clock until the surfaces of the spheres with the radii $\rho_{1}$, $\rho_{2}, \rho_{3}$ and $\rho_{4}$ all intersect each other at one point (Figure 2). For single point positioning, in other words, four satellites must be in view at one time. (Three satellites will suffice when the altitude is fixed and only the latitude and longitude are being sought.)

The positioning precision is affected by the positions of the satellites at the time of observation. The level of this effect is defined as the square root of the sum of the diagonal elements in the weighting matrix for the positioning calculation and is called the GDOP (geometrical dilution of precision). The GDOP may be divided into the part related to position or PDOP and that related to time or TDOP. The PDOP may be further subdivided into the vertical component (VDOP) and the horizontal component (HDOP). Under normal circumstances, observation will be possible if the PDOP is below 6 and the observation conditions considered good if it is below 3 .

\section{POSITION INDICATION SYSTEM USING GPS NAVIGATION SENSORS}

\subsection{GPS Navigation Sensors}

The navigation sensors used in the study were the TANS produced by Trimble Navigation Ltd. On the TANS, the C/A codes on the $\mathrm{L} 1$ band waves transmitted from the satellites are received sequentially by its two channels.

Data on such items as the position and velocity are provided from the TANS through two-circuit RS-422 interface and application systems suited to the needs of the user can be created by connection to a personal computer. The principal specifications of the TANS are given in Table 1.

\subsection{Composition of Position Indication System}

The system developed under this study consists of the GPS navigation sensor (TANS), aerial and portable personal computer (NEC PC98NOTE) (Photograph 1).

The software part consists of the data retrieval from the sensor and the data display including the locus charts. The TANS and the personal computer are linked by the two-way communication cable RS-422 and the TANS can be made to 
output the required data by sending signals from the personal computer.

The data display consists of the initialisation, positioning, locus plotting and system data displays.

\subsection{Tests for Confirmation of Operational Characteristics}

The position indication system was used in tests to confirm the operational characteristics of the TANS. The tests were carried out for single-point positioning, in which the position was measured on a single position indication system, and for differential positioning, in which two systems were used, one as the fixed station and the other as the mobile station, and the difference between the positioning results obtained on the two systems was used. It is said that differential positioning will raise the accuracy of the measurement because the systematic errors, such as those caused by the transmission delay in the ionosphere and atmosphere, are cancelled out by the simultaneous observation using two systems. It has been reported that the error can be reduced to within 3 m by differential positioning. ${ }^{31}$

\section{(1) Test Conditions}

The position indication system was installed on a vehicle and measurements made while travelling in a circle with a $20 \mathrm{~m}$ radius. A fixed station was set up at the centre of the circle and measurements made here at the same time as the mobile station. Radio waves were received from four satellites (No. 12, 20, 21 and 23) and the PDOP was between 4.2 and 6.8. The total measurement time was approximately 25 minutes and the travelling speed of the mobile station approximately $4 \mathrm{~km} / \mathrm{h}$.

\section{(2) Single-Point Positioning}

The results of the measurements made while travelling five times around the circle are shown in Figure 3. It is to be observed that the loci move towards the southwest with time. Between the first and the last circles, the position of the centre of the circle (average latitude and longitude of the loci) moves as much as $11 \mathrm{~m}$ southwestwards. The distances of the points on the loci from the centres of the circles were calculated. The RMS errors from the radius of $20 \mathrm{~m}$ were between $1.0 \mathrm{~m}$ and $3.2 \mathrm{~m}$, indicating that, although there is a systematic variation of around $10 \mathrm{~m}$, the relative positions within short periods of time were well maintained in single-point positioning from moving bodies.

\section{(3) Differential Positioning}

The results obtained through differential processing at 1 second intervals are shown in Figure 4. The systematic error observed in single-point positioning is eliminated by the differential processing but the precision regarding the positions relative to the centres of the circles is lowered. The RMS errors of the distances between points on the loci and the fixed point for each circle in relation to the $20 \mathrm{~m}$ radius ranged between 2.1 and $3.2 \mathrm{~m}$. These are greater than the errors found in single-point positioning, indicating lowering of the precision regarding relative positions over short periods of time.

Since the relative positions over short periods of time were well maintained, the values obtained by averaging the measurement positions of the fixed point over constant time intervals were subtracted from the single-point positioning results. The averaging time here was the time it took to make one circle (100 seconds) (Figure 5 ). In the results thus obtained, the systematic error observed in the single-point positioning has been eliminated, while RMS errors for distances of the points on each circle from their centres show 
relatively low values of between 1.3 and $2.6 \mathrm{~m}$, indicating that the relative positions too are well maintained.

\section{APPLICATION TO TRAVELLING VIBRATORY ROLLERS}

The system was installed on a vibratory roller and loci obtained for when the roller was made to travel to and fro (10 times) over a straight section $80 \mathrm{~m}$ in length were indicated (Photograph 2). A fixed station was set up at the end of the travel path for simultaneous measurement. The satellites used were No. $12,20,21$ and 23 for the first eight passes and No. 3, 12, 20 and 21 for the last two passes. The PDOP ranged between 3.2 and 3.5 . The total observation time was 14 minutes.

\section{(1) Single-Point Positioning (Figure 6)}

The locus for each pass tend to be linear, but the loci as a whole are seen to move towards the northwest with time. The centres of the loci for sets of two passes each were obtained in terms of the average of the coordinates of all points on the loci. There is a distance of $16.7 \mathrm{~m}$ between the centre of the first two passes and the last two, giving a systematic error greater than that observed in the test for confirmation of operational characteristics.

\section{(2) Differential Positioning}

Since the results obtained by differential processing at 1 second intervals were unsatisfactory, the coordinates obtained by averaging out the measurement positions for the fixed station at travel time zones for each two passes were subtracted from the measurement coordinates for the mobile station (Figure 7 ). By doing so, the systematic errors observed in the single-point positioning for the first eight of the ten passes were eliminated. The RMS errors for the distances of each point from the average travel path was reduced to $2.0 \mathrm{~m}$.

The systematic errors, however, remain for the last two passes. This is because of the change in the combination of satellites used and the discrepancies in the measurement results cannot be eliminated simply by taking away the differences in measurement results at the mobile and fixed stations. In order to eliminate this systematic error, one needs to correct separately the pseudo ranges from each satellite to the sensor before repeating the positioning calculations. Since the system is incapable of outputting pseudo ranges, this method cannot be used at present.

The system, as it stands, is set so that it will select the combination of satellites that will give the smallest PDOP. The error resulting from changes in the combination of satellites can be reduced by altering the software so that the system will stay tuned to the same combination of satellites, regardless of the PDOP, until one of the satellites goes out of range.

\section{CONCLUSION}

A position indication system that makes use of GPS navigation sensors was developed and investigations made on the application of this system to tracking of heavy earthwork equipment.

The characteristics of the system are as follows.

1) The system can be used in any place whence it is possible to observe the GPS satellites.

2) The system is capable of indicating the measurement positions in real time (at 1 second intervals).

3) The precision of the positioning may be raised by implementing differential processing using two position indication systems. 
As regards item 3 ), because of the low degree of precision obtained in processing at 1 second intervals, the values obtained by averaging the measurement results at the fixed stations over constant intervals $(60$ seconds or over) are used instead.

The points of note regarding the application of the system to positioning of earthwork equipment are as follows.

a) The system can be applied to rough tracking of operating position of the equipment by single-point positioning.

b) The system can be applied to the level for tracking travel paths of moving equipment, by subtracting the average position of the fixed station at constant time intervals from the measurement positions of the mobile station as a means of differential processing, and so raising the degree of precision.

The differential process here, however, is a simplified process in which the differences between the measurement results for the mobile and fixed stations are subtracted and has the problem that the systematic error cannot be eliminated in this way when there is alteration in the combination of satellites used for the positioning calculations.

The authors hope in the future to continue their research on communication systems between the mobile and fixed stations and on measures for dealing with the changes in the combination of satellites used for positioning. They hope also to expand the scope of the system through combination of the navigation sensors with other types of sensors.

\section{REFERENCES}

1) Shunsuke Sakurai, Norikazu Shimizu: Applicability of Global Positioning System to Displacement Measurements in Rock Engineering, Proceedings of the 23rd Symposium on Rock Mechanics, 1991.2.

2) Yoshifumi Nakagawa, Tomonori Takada, Tatsunori Sada: Study on the Application of GPS to Site Management, Proceedings of the 8th Research Presentation and Discussion Meeting on Construction Management, 1990.12.

3) Peter Byman, Ilari Koskelo: MAPPING FINNISH ROADS WITH DIFFERENTIAL GPS AND DEAD RECKONING, GPS WORLD, 1991.2. 


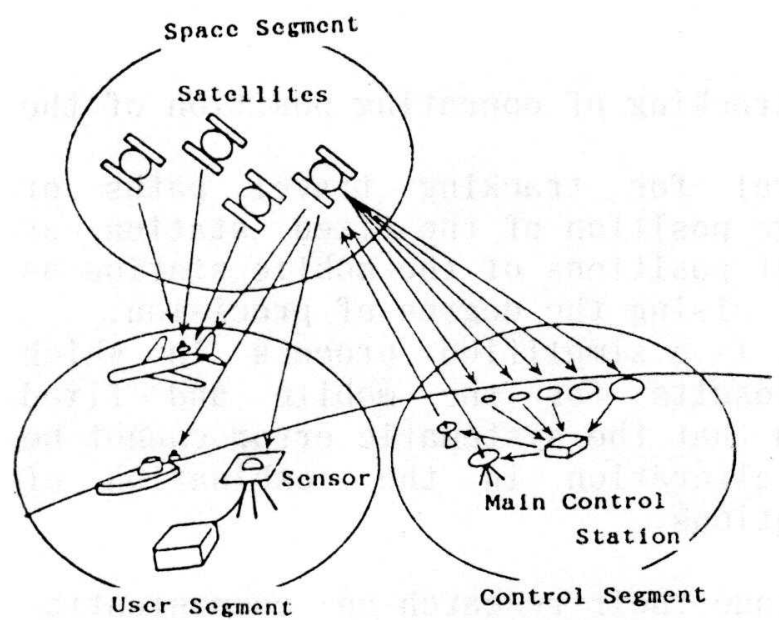

Figure 1 Composition of GPS

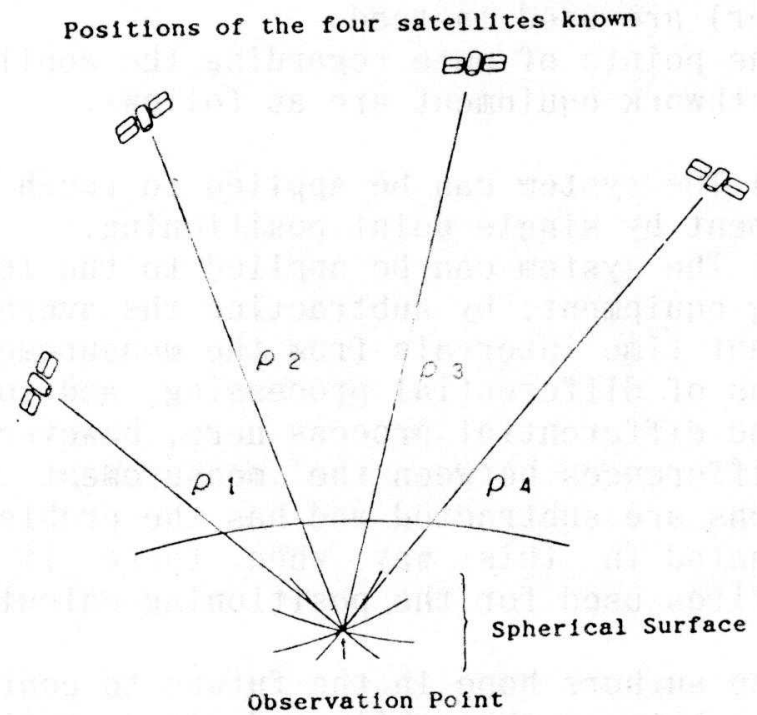

Figure 2 Principles of Single-Point Positioning

Table 1 Principal Specifications of TANS

\begin{tabular}{|c|c|}
\hline Iter & Specification \\
\hline Sensor & $\begin{array}{l}\text { L1 frequency, C/A code, } 2 \text { channel } \\
\text { sequential reception }\end{array}$ \\
\hline Positioning Mode & $\begin{array}{l}\text { 1) } 4 \text { satellites: } 3 D \text { positioning } \\
\text { 2) satellites: 2D positioning } \\
\text { (fixed altitude) } \\
\text { 3) Automatic: } 30 \text { when } 4 \text { satellites } \\
\text { within range } \\
\text { 2D when only } 3 \text { sate- } \\
\text { llites within range }\end{array}$ \\
\hline $\begin{array}{l}\text { Horizontal Position } \\
\text { Precision }\end{array}$ & 25 - (SEP) \\
\hline $\begin{array}{l}\text { Vertical Position } \\
\text { Precision }\end{array}$ & 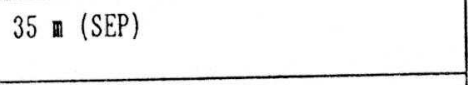 \\
\hline Velocity Precision & $0.2 \mathrm{~m} / \mathrm{s}$ (RMS) at constant speed \\
\hline Time Precision & within 1 micro-second of UTC \\
\hline $\begin{array}{l}\text { Positional } \\
\text { Dispersion } \\
\end{array}$ & $\begin{array}{l}5 \text { (RYS) or under per } 10 \text { minutes } \\
\text { when PDOP }<8\end{array}$ \\
\hline $\begin{array}{l}\text { Position Renewal } \\
\text { Time }\end{array}$ & 1 second \\
\hline Digital Interface & 9600 baud 2-way RS-422, 2 channels \\
\hline Dimensions & 127 m $\times 241$ m $\times 50$ m \\
\hline Weight & $1.27 \mathrm{~kg}$ \\
\hline Power Consumption & 9 to $32 \mathrm{VDC}, 4 \mathrm{~W}$ of less \\
\hline
\end{tabular}

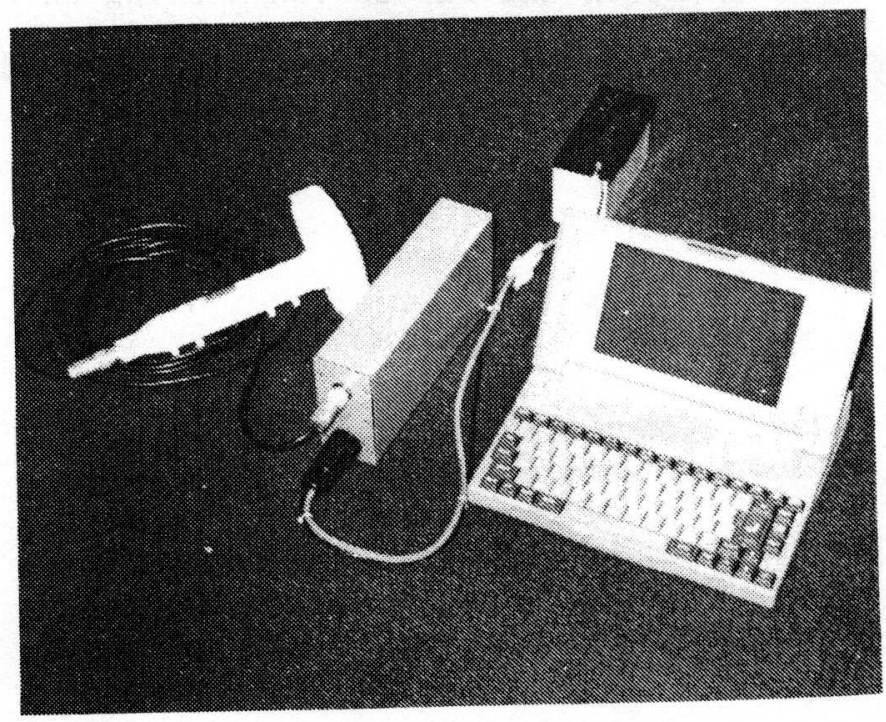

Photograph 1 System Composition 


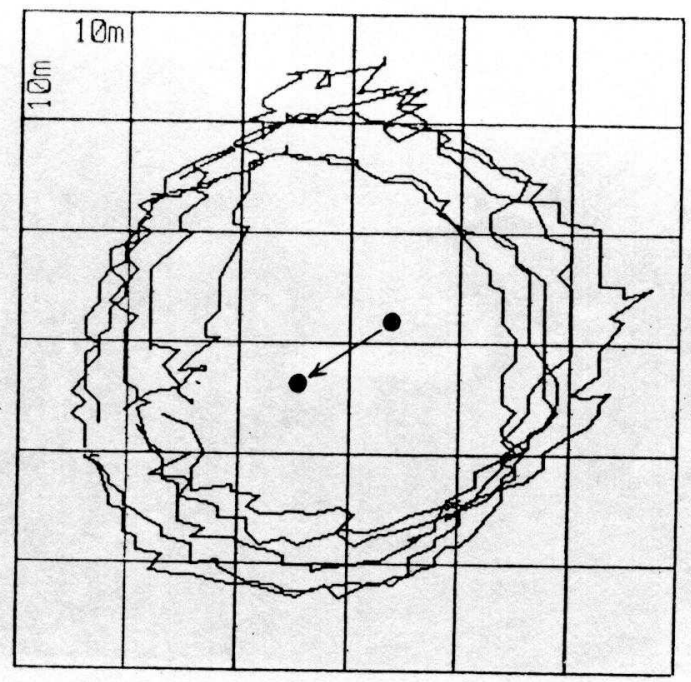

Figure 3 Single-Point Positioning Results

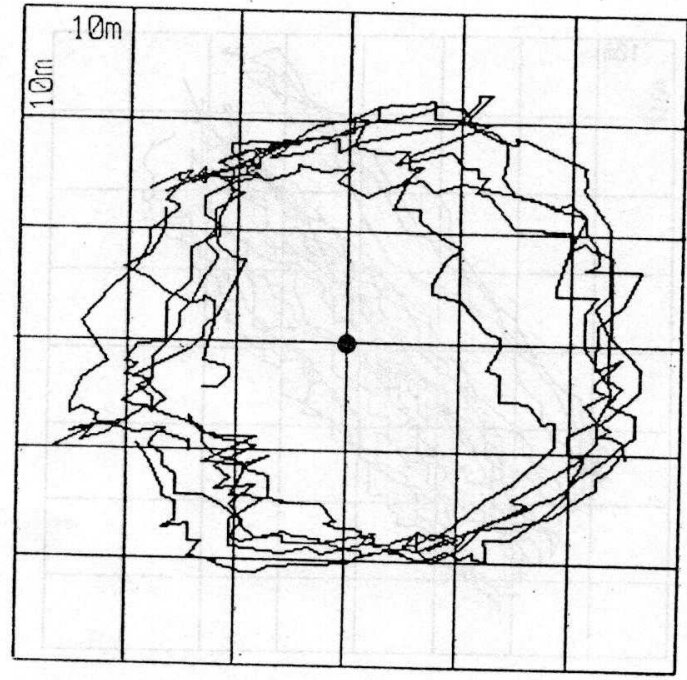

Figure 4 Differential Positioning Results (Unit Time: $1 \mathrm{sec}$ )

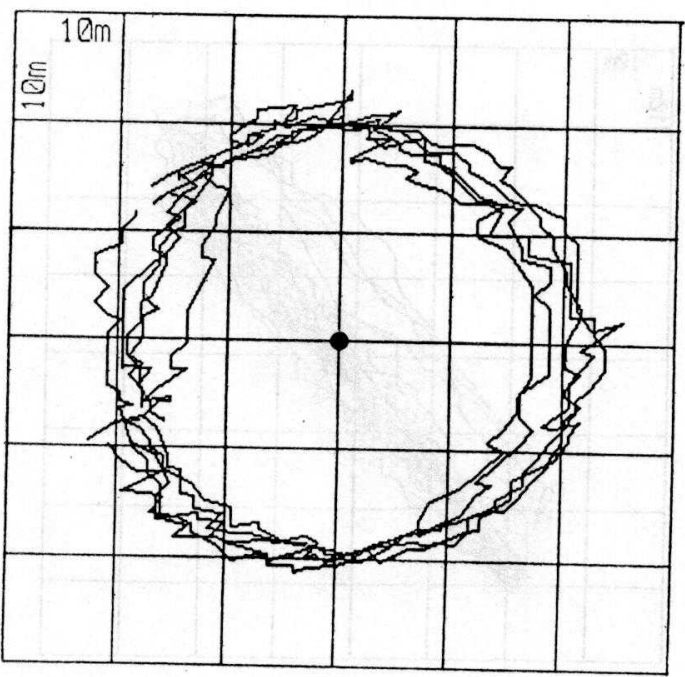

Figure 5 Average Differential Processing Results 


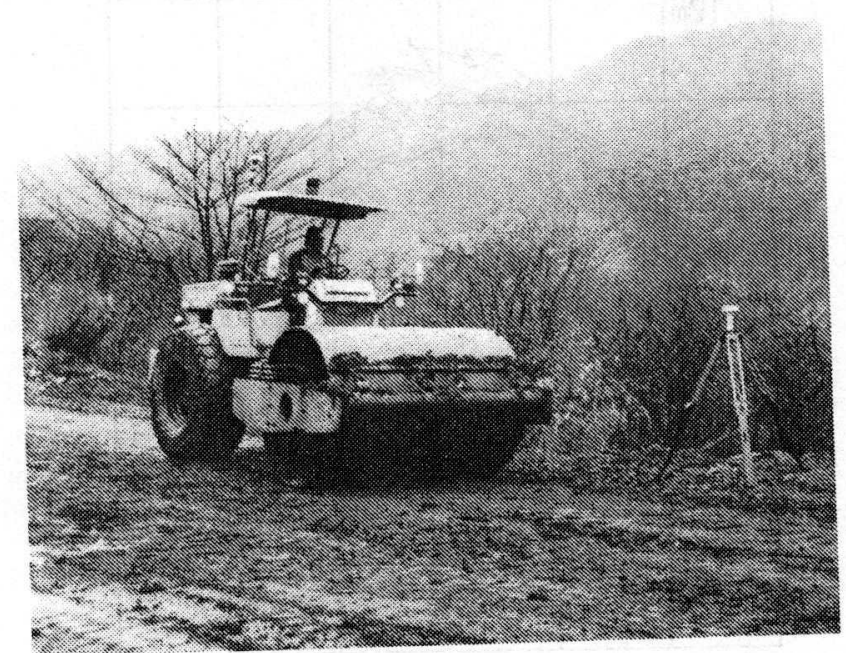

Photograph 2 Travelling Test Using Vibratory Roller

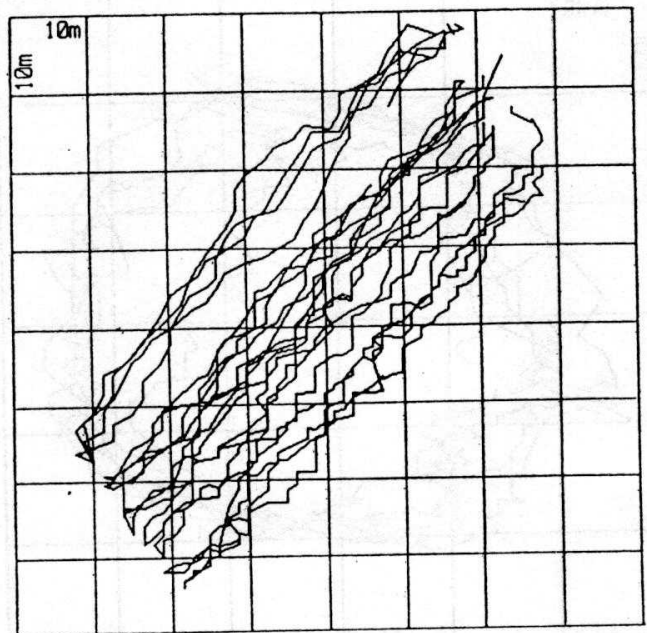

Figure 6 Single-Point Positioning Results

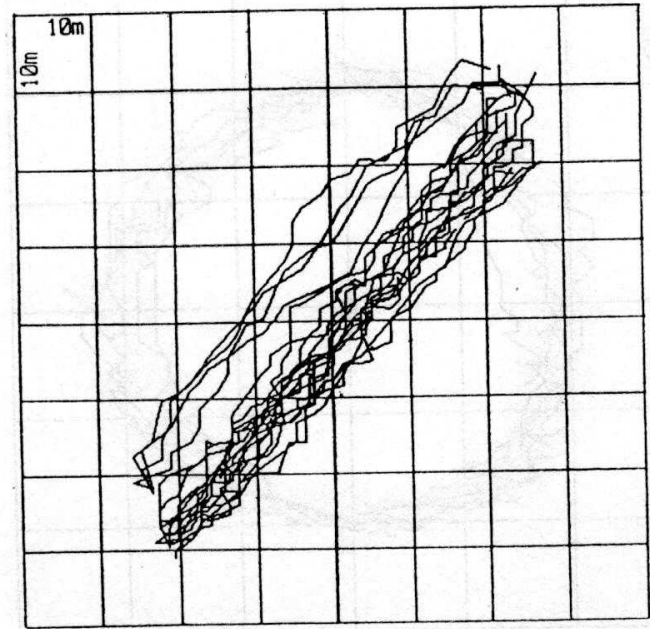

Figure 7 Results of Differential Processing by Averages 